\title{
Racismo y segregación en colombia: salud, educación y trabajo en la población afrodescendiente del pacífico'
}

Jimara Martínez

Dayrina Pomares

dayrinap@uninorte.edu.co
Mauricio Sierra amauricios@uninorte.edu.co

María G. Martínez gmartinezm@uninorte.edu.co

\section{Universidad del Norte- Departamento de Ciencia Política y Relaciones Internacionales Barranquilla, Colombia - 2020}

Envío recibido 2020-06-20 aprobado 2020-12-23

\section{Cómo citar este artículo:}

Martínez Tovar, J.A., Pomares, D., Sierra, M., \& Martínez, M. G. (2021). RACISMO Y SEGREGACIÓN EN COLOMBIA: SALUD, EDUCACIÓN Y TRABAJO EN LA POBLACIÓN AFRODESCENDIENTE DEL PACÍFICO. Trans-Pasando Fronteras, (16). https://doi.org/10.18046/retf.i16.4102

\footnotetext{
${ }^{1}$ El presente artículo de revisión es resultado de una investigación que lleva el mismo nombre (Racismo y Segregación en Colombia: Salud, Educación y Trabajo en la población Afrodescendiente del Pacífico), desarrollada en el marco de la clase de Seminario de Investigación del programa de Relaciones Internacionales (RRII) de la Universidad del Norte. Así mismo, esta investigación se origina con el fin de aportar información sobre la situación de la población afrodescendiente del Pacífico colombiano en materia de salud, educación y trabajo e influir en la necesidad de tomar acciones que mejoren su calidad de vida.
} 


\section{Resumen}

Este artículo busca analizar las evidencias encontradas por medio de una revisión bibliográfica, acerca de la manifestación de la segregación racial y vulnerabilidad de los afrodescendientes del pacífico colombiano en las áreas de empleo, salud y educación. Así mismo, se contrastan los datos encontrados en cada una de las áreas con lo planteado en las teorías de Derechos Humanos y la teoría de Centro-Periferia que se analizan a lo largo del artículo. Lo anterior, en pro de lograr un mejor entendimiento sobre cómo el racismo afecta al desarrollo pleno de estos derechos humanos para esta comunidad, y exponer las dinámicas diferenciales que experimenta el Pacífico como periferia frente a las grandes ciudades del país como centros. Finalmente, se exponen las conclusiones sobre la relación existente entre el color de la piel y la ubicación geográfica con la calidad de vida, el desarrollo socioeconómico y el cumplimiento de los derechos humanos de esta población.

Palabras clave: Racismo, Afrocolombiano, Derechos Humanos, Pacífico Colombiano, Desigualdad. 


\title{
Racism and segregation in colombia: health, education and work in the afro-descendent population of the pacific
}

\begin{abstract}
This article seeks to analyze the evidence found through a bibliographic review, about the manifestation of racial segregation and vulnerability of Afro-descendants of the Colombian Pacific in the areas of employment, health and education. Likewise, the data and informationfoundineach of theareasarecontrasted withwhatisstated in the theories of Human Rights and the Center-Periphery theory that are analyzed throughout the article. The foregoing, to achieve a better understanding of how racism affects the full development of these human rights for this community, and to expose the differential dynamics that the Pacific experiences as a periphery compared to the country's large cities as centers. Finally, the conclusions on the relationship between skin color and geographic location with quality of life, socio-economic development and compliance with the human rights of this population are presented.
\end{abstract}

Keywords: Racism, Afro-Colombian, Human Rights, Colombian Pacific, Inequality. 


\section{Introducción}

La región pacífica colombiana se encuentra ubicada al occidente del país y está conformada por los departamentos del Chocó, Valle, Cauca y Nariño. Es uno de los territorios más afectados por el conflicto interno y su población está compuesta mayoritariamente por comunidades afrocolombianas y pueblos indígenas. Para el censo del DANE del 2018, la población actual perteneciente a las comunidades afrocolombianasnegras, palenqueras y raizales- equivale a 2.982.224 millones de personas, sin embargo, esta cifra se ha reducido en un 30,8\% desde el censo del 2005, que había contabilizado un total de 4.311.757. A partir de 1980, las comunidades afrocolombianas empezaron movimientos en pro del reconocimiento de tierras como colectivas, pero esto solo se pudo solidificar con el reconocimiento de su carácter de grupo étnico en la Constitución de 1991, el artículo 55 transitorio y la posterior expedición de Ley 70 de 1993 que otorgó el reconocimiento de sus tierras como colectivas, ya que antes del 91 no eran comunidades reconocidas (Ayala, Flórez, Hurtado y Lobato, 2014). Sin embargo, aún en la actualidad, estas comunidades siguen siendo vulnerables y víctimas de expropiaciones, desplazamientos y violaciones de sus derechos humanos.

En este sentido, la situación del pacífico colombiano tiene una agravante; la segregación ha sido sistemática. Como lo anota el informe sobre la problemática humanitaria del Pacífico colombiano realizado por la Defensoría del Pueblo (2016), la región ha sido vista históricamente como "un territorio marginal o de frontera agrícola, con una serie de características geográficas (topográficas, de acceso y climáticas), que obstaculizan o dificultan sus posibilidades de desarrollo social, crecimiento económico e integración cultural y política" (p.36). Estas nociones continuaron durante la época republicana; a pesar de que hubo ciertas reformas económicas y políticas, la visión cultural no 
sufrió transformaciones. Por lo tanto, el territorio, como su población, debían ser domesticadas a través de la violencia y la esclavitud, cuestión que en la actualidad se transformó en connotaciones de discriminación y racismo (Defensoría del pueblo, 2016).

El objetivo general de este artículo es analizar las evidencias de la segregación hacia la población afrodescendiente del pacífico colombiano. Se enlazará la teoría de los derechos humanos y centroperiferia con las realidades vividas por los afrocolombianos del pacífico colombiano en los ejes ya mencionados. Se debe tener en cuenta, que la teoría de los derechos humanos es reconocida como un objeto de estudio más que una teoría como tal. Sin embargo, esta teoría pretende exponer la historia de estos, cuáles derechos humanos existen, su importancia y cómo hacerlos valer. Así mismo, esta teoría es analizada y tomada por varios autores para explicar problemáticas locales o internacionales con respecto a la violación de los mismos. Por su parte, la teoría de centro-periferia, según Gunder, Cockcroft \& Johnson (1968), describe cómo las metrópolis (el centro) acaparan los recursos producidos por los satélites (periferias) para su propio desarrollo. Es decir, las grandes ciudades o centros urbanos de un país tienden a desarrollarse más, debido a que la población o la mano de obra que sustenta la economía tiende a movilizarse a lugares donde pueden obtener mejores ingresos, pero estas movilizaciones repercuten en que las periferias vivan en un constante subdesarrollo o crezcan a menor velocidad que los centros o metrópolis.

Con base en las teorías, se analizará también, los problemas a los cualesseenfrentala poblaciónafrodescendientedel pacíficocolombiano en cuanto a sus derechos humanos. Lo anterior para demostrar que es una realidad constante que merece mayor atención no solo por parte de los gobiernos locales y el gobierno nacional, sino también por parte de los investigadores y académicos a manera de estudio. Así mismo, se pretende generar literatura que contribuya a identificar las causas y consecuencias de la violación de los derechos humanos. En este marco, 
se entenderán los derechos humanos a la educación, el trabajo y la salud, como principales motores de la consecución de una vida digna y un pleno desarrollo socioeconómico.

Acontinuación, se presentarála metodología base de la investigación, en la cual se detallan las fuentes utilizadas, el tipo de investigación y otras especificidades. Posteriormente, se presentará y se presentará la discusión, donde se le dará desarrollo a los ejes principales de la investigación: en el primero de estos ejes, se analizarán las barreras existentes al momento de acceder al mercado laboral fuera de la región pacífica para los afrodescendientes; el segundo eje se centra en la desigualdad existente en cuanto a la calidad del sistema de salud al que tienen acceso la población afro del pacífico colombiano con respecto a otras zonas del país; y por último, en el tercer eje se discutirá sobre la calidad de la educación a la que pueden acceder la población afro del pacífico con respecto a otras regiones del país. Finalmente, se abordarán las conclusiones a las cuales se llegaron una vez realizado el análisis de la información obtenida a través de la bibliografía, el diálogo con los autores de esta bibliografía y el entrelazamiento entre la teoría y las realidades vivenciadas por los miembros de la comunidad afro del pacífico colombiano.

\section{Metodología}

Para conocer sobre la segregación racial y la violación de los DDHH en la población afrodescendiente en Colombia, se realizará una revisión de literatura en donde se desarrollarán fichas bibliográficas de diferentes aspectos concernientes a esta temática, es decir, se hará una investigación de tipo secundaria. Según De Dios y Álvarez (2006), estas investigaciones "son aquellas en que no es necesario realizar la valoración crítica de los documentos, pues otros compañeros (generalmente expertos en esa materia) ya lo han realizado por nosotros". Lo anterior, con el fin de observar la variedad de puntos de vista y métodos que diferentes autores utilizaron para llevar a cabo 
estudios con respecto a la problemática. La literatura revisada estuvo encaminada a cubrir las áreas de salud, educación y empleo en el pacífico colombiano. De esta manera, se realizó un estudio descriptivo de la situación de esta región y su diferencia con las dinámicas en el resto de país, tratando de encontrar evidencias que reflejaran la dicotomía en el cumplimiento de los derechos humanos.

De igual forma, se tuvieron en cuenta estudios e investigaciones cualitativas y cuantitativas realizadas en la región con respecto al desarrollo de enfermedades, percepción del acceso a la educación o capacidad de acceder a empleos. A partir de esto, se hizo un contraste de estas realidades con lo planteado en las teorías utilizadas para explicar el fenómeno de los Derechos Humanos en el pacífico; la teoría de Derechos Humanos y la teoría Centro-Periferia.

Así mismo, se realizará un análisis por medio del cual se describirán los diferentes fenómenos que afectan a la comunidad afrodescendiente con respecto a los no afrodescendientes. Según Dankhe (1986) citado en (Hernández Sampieri et al., 1997, p.71), los estudios descriptivos buscan desarrollar una descripción del fenómeno estudiado a partir de sus características, midiendo conceptos con el fin de especificar las propiedades importantes de personas, comunidades o grupos bajo análisis. Por otro lado, con el fin de obtener unos resultados más amplios y profundos, se pretende utilizar el método cualitativo, el cual Monje (2011) indica que:

"Se nutre de la hermenéutica, la fenomenología y el interaccionismo simbólico [...], se interesa por la necesidad de comprender el significado de los fenómenos y no solamente de explicarlos en términos de causalidad [...], el conocimiento está mediado por las características sociales y personales del observador; que no existe una realidad exterior al sujeto [...], la realidad de los individuos se estudia desde el interior, a partir de lo que ellos perciben a través de sus experiencias vividas" -Monje. 2011. p. 12-13. 


\section{Discusión}

La población negrasiempre hasido víctima de segregación, exclusión, violencia y otros problemas que conlleva el racismo. "Las mujeres y los no blancos fueron, hasta bien entrado el siglo XX, comúnmente considerados en Occidente como irremediablemente deficientes en sus capacidades racionales o morales y, por lo tanto, incapaces de ejercer sus derechos humanos" (Donelly, 2003, p. 169). Para contrarrestar esto, Colombia ha buscado la protección de las comunidades negras y promover su inclusión. Con la ley 70 de 1993, en la cual se desarrolla el artículo transitorio 55 de la Constitución Política, se pretende "establecer mecanismos para la protección de la identidad cultural y de los derechos de las comunidades negras de Colombia como grupo étnico, y el fomento de su desarrollo económico y social". Esto con el fin de garantizar a las comunidades negras condiciones justas de igualdad de oportunidades frente al resto de la población.

Sin embargo, si bien el país le ha apostado a mejorar las condiciones de vida y el entorno de las comunidades afrocolombianas -negras, palanqueras y raizales-, la realidad vivida por estas poblaciones permeada por la exclusión, marginalización, mala calidad de vida y omisión estatal, lleva a analizar y criticar el hecho de que no solo basta estar escrito en el papel, sino que también son necesarias medidas y políticas públicas como acciones afirmativas de mejor calidad y profundidad acordes a estas realidades geográficas, socioculturales, políticas y económicas.

\section{Obstáculos a los que se enfrenta la población afrodescendiente del Pacífico colombiano en el ámbito laboral.}

El racismo, la segregación y la falta de garantías de los derechos humanos, es evidentey radicavarios sectores de lasociedad colombiana, como la salud, la educación y las oportunidades de trabajo. Según Marulanda (2014), "existen brechas salariales entre afrodescendientes 
y no afrodescendientes con la misma ocupación y nivel de preparación, siendo los afrodescendientes los que ganan menos que los demás trabajadores" (p. 323).

Marulanda y Rodríguez (2015), a través de la construcción de un índice de la calidad del trabajo, lograron identificar las desigualdades existentes entre personas pertenecientes a poblaciones afro y no afro, particularmente, en la ciudad de Cali. Estas desigualdades se encuentran especialmente en los salarios. "Para la población no afro el salario promedio es de \$947.692 [pesos], mientras que para los afrodescendientes el promedio salarial es $\$ 770.900$ [pesos]" (Marulanda y Rodríguez, 2015, p. 335).

Así mismo, las personas negras o las personas que componen y hacen parte de comunidades negras, son más propensas a ser parte de la población desempleada o informalmente empleada en el país, por razones comúnmente asociadas al color de piel o la posición socioeconómica que poseen. "Ser afrodescendiente reduce las probabilidades de tener empleos de media y alta calidad en alrededor del 1,8\% y $1 \%$ e incrementa la posibilidad de tener un empleo de mala calidad en 2,8\%" (Marulanda y Rodríguez, 2015, p.340).

Como último dato con respecto a este estudio realizado por Marulanda y Rodríguez (2015), está el hecho de que, según los resultados expuestos, la estabilidad laboral que presentan las personas pertenecientes a las comunidades afro es de 13,70\%, mientras que la estabilidad laboral que tiene el resto de la población es de 53,90\%. Lo anterior, permite denotar con mucha facilidad la gran brecha existente en el ámbito laboral entre ambos grupos, y más si se tiene en cuenta la importancia que tiene para cualquier persona poder contar con un empleo y que este sea estable. Estos resultados se aproximan a los obtenidos en un estudio realizado por Arroyo y Bustamante (2008) en Cali: 
"Una persona denominada negra tiene mayor probabilidad de encontrarse en un empleo de mala calidad, cuyo efecto sobre esta última es del 12,2\%. Siguiendo una tendencia similar, el ser un trabajador negro reduce las probabilidades de acceder a empleos de mejor calidad, reducción que es de 6,9\%, 2,1\% y 1,1\% para los empleos de calidad media inferior, media superior y buena calidad" -(Arroyo y Bustamante, 2008, p. 159).

Estos autores revelan en su estudio que esta tendencia no solo se encuentra en la ciudad de Cali, sino también en el resto del territorio nacional:

"Al contrastar estos efectos con los estimados para Cali se puede observar que el ser negro a nivel nacional tiene un mayor efecto en las probabilidades de que tal trabajador tenga un empleo de mala calidad, siendo este de 14,4\%, es decir 2,2\% más alto. (...) Esto se evidencia en que los efectos marginales para los empleos de calidad media inferior, media superior y buena calidad son muy similares para Cali y para Colombia; no obstante, estos son un poco mayores para el total de las trece áreas metropolitanas (...) Lo anterior apoya la idea de que a nivel nacional hay una mayor discriminación laboral asociada con la calidad del empleo de las personas negras" -(Arroyo y Bustamante, 2008, p. 159-160).

Lo anterior, crea un círculo vicioso que involucra bajos niveles de crecimiento económico con bajos niveles de ahorro, y en consecuencia bajos niveles de educación con bajos niveles de capital humano; lo cual resulta en una baja productividad que no permite un óptimo desarrollo en la vida laboral, con una agravante si se tiene en cuenta el factor de desigualdad con respecto a otras poblaciones del país. Además, Para el segundo semestre de 2019, según el boletín técnico del DANE (2019) de la Gran Encuesta Integrada de Hogares (GEIH), la región pacífica ocupaba el tercer lugar con mayor tasa de desempleo y el total de la población desocupada fue de 460 mil personas.

Las investigaciones y los datos descritos en los párrafos anteriores, sumado a las teorías que se han escogido para desarrollar el marco 
teórico referentes a los derechos humanos, evidencian y soportan las ideas de que, en definitiva, ser negro o negra en una sociedad como la colombiana, significa ser más vulnerable económica, social, política y culturalmente. Lo anterior, es manifestado por Goodale y Merry (2007) por medio de las explicaciones de tipo ideológico, sociológico y cultural que se refieren a patrones de comportamiento que generan discriminación y normalizan la permanencia de patrones de odio entre comunidades. En la actualidad, Ios Objetivos de Desarrollo Sostenible (2018), reconocen que existe una mayor incidencia de pobreza y pobreza extrema en las comunidades indígenas y afrodescendientes que en las otras comunidades sin identidad étnica o racial (Correa, 2018). Así mismo, Rangel y Del Popolo (2011) también reconocen en sus investigaciones que los afrodescendientes se encuentran con condiciones de exclusión, desigualdad y alto desempleo.

Por otro lado, los afrodescendientes se ven obligados en muchos casos a abandonar sus lugares de origen para trabajar en grandes metrópolis en busca de mejores oportunidades. Las razones de estas dinámicas migratorias son en su mayoría debido a problemas de desigualdad social y económica que se agudizan con las problemáticas de la región como desplazamiento forzado, recrudecimiento de la violencia y falta de oportunidades que inciden a su vez en que se violen los derechos humanos, y para motivos de esta investigación, el derecho al trabajo. Lo anterior, es explicado por Risse-Kappen, Risse, Ropp y Sikkink (1999) referentes a la teoría de los derechos humanos, los cuales argumentan que existen factores de tipo económico que explican cómo el desarrollo económico y la distribución del capital en las sociedades perpetúan sistemas de desigualdad.

De igual forma, el Estudio de Población llevado a cabo en 1981 por el Departamento Administrativo Nacional de Estadísticas (DANE) en Medellín, Antioquía, puede servir de base para entender la posición de los chocoanos en la ciudad y las dinámicas que han sido desarrolladas desde tiempo coloniales quedando imperantes en la sociedad colom- 
biana. La muestra del censo era del 10\% de la población de Medellín y captó 712 personas chocoanas. De estos, 670 tenían más de doce años y 415 tenían empleo. Las mujeres superaban a los hombres en la proporción y eran el 60\% de los chocoanos con empleo (Wade, 1993).

En este sentido, los autores Gunder, Cockcroft y Johnson (1968), describen cómo las metrópolis (el centro) acaparan los recursos producidos por los satélites (periferias) y las personas de estos satélites para su propio desarrollo. Respecto a esto, la población de las periferias o satélites del pacífico, son explotadas o se trasladan a los centros como Medellín u otros departamentos más desarrollados para conseguir mejores oportunidades de empleo - aun si son empleos de carácter informal-, lo que genera un enriquecimiento por parte de los segundos y un deterioro en los territorios periféricos o un crecimiento más lento.

Respecto a la dinámica Chocó-Multinacionales, -entendiéndose Chocó como periferia y multinacionales como centros- en estas relaciones los segundos extraen los recursos del territorio chocoano bajo modalidades de explotación directa empleando trabajadores con pagos muy bajos y de explotación indirecta en la cual se intercambian estos recursos por artículos manufacturados de toda clase y/o comida (Wade, 1993), reproduciendo y replicando así vínculos por los cuales el sistema nacional e internacional absorbe los recursos naturales de la región y la teoría centro-periferia toma fuerza. Un ejemplo de esto es la industria minera y lo que conlleva para la región; según un informe de la Organización Internacional para las Migraciones (2015) en este territorio existen grandes multinacionales que controlan la minería, estas a su vez explotan laboralmente a las poblaciones y trabajadores, $y$, por consiguiente, convierten este trabajo en una fuente de ingreso inviable. Así mismo, la explotación minera ha intensificado las confrontaciones entre grupos armados por el control de este, ya que no está correctamente organizada, democratizada o legalizada, y como consecuencia genera necesidades básicas insatisfechas y nuevas olas de desplazamiento hacia centros urbanos. 
En este marco, también es importante resaltar que el tipo de trabajo que hace la población de la periferia en su mayoría es de carácter informal. Según el informe del Banco de la República sobre informalidad en el Pacífico (2015), por sector económico, se encuentra en primer lugar ser empleado doméstico, seguido de trabajar por cuenta propia y en tercer lugar ser patrón o empleador. Lo anterior explica que exista "una distribución centro-periferia en donde la población de los barrios perimetrales son los que más parecen enfrentar el fenómeno de la informalidad".

Así mismo, el proceso de obtención de un trabajo está compuesto por varias fases previas donde el candidato debe cumplir con ciertos requisitos durante varias etapas. Las bajas probabilidades de conseguir trabajos de alta calidad que tienen los miembros de las comunidades afro se explican, en gran parte, porque en el proceso de selección, también se ve permeado por la discriminación racial (Cárdenas, Rodríguez, Oviedo y Villamizar, 2013). En esta sección, nuevamente se hace relevante lo expresado por Goodale y Merry (2007) sobre los factores de tipo ideológico, sociológico y cultural que generan discriminación y normalizan la permanencia de estas, pero también se hace necesario incluir los factores de tipo económico de Risse-Kappen, et al. (1999), los cuales hablan de cómo el desarrollo económico y la distribución del capital en las sociedades perpetúan sistemas de desigualdad.

El informe del Banco de la República (2015) también destaca la existencia de grupos o "clubes" de informalidad en la región Pacífica, en la cual, superar los niveles de educación escolar y por menos estudiar un técnico reduce las probabilidades de enfrentar trabajos informales y conseguir empleos de mejor calidad. En este sentido, en la ciudad de Bogotá, se evidenció que los candidatos de descendencia afro son quienes menos reciben llamadas a entrevistas de empleo:

"En primer lugar, las hojas de vida de afrodescendientes reducen en un $7,79 \%$ la probabilidad de una llamada si se comparan con el resto de las hojas de vida, mientras que las de personas blancas aumentan 
la misma probabilidad en 3\%. [Por ende] los aspirantes afrodescendientes son quienes reciben menos llamadas a entrevistas de empleo" -(Cárdenas et al., 2013, p. 19).

\section{Segregación en el acceso y calidad de la salud de la población afrodescendiente del Pacífico colombiano.}

La población afrodescendiente en la región del pacífico se encuentra entre las comunidades más vulnerables y excluidas como consecuencia del racismo estructural presente en la sociedad colombiana. El racismo se encuentra inscrito en muchas áreas o momentos de la vida en sociedad, en cuestiones económicas, políticas y sociales, generando dinámicas de pobreza y exclusión. Así, la discriminación que enfrentan los afrodescendientes del pacífico al momento de acceder al sistema de salud no es directa, pues esta no se basa en características físicas como el color de la piel; sino más bien en una configuración de la estructura del sistema social. Debido a la falta de presencia estatal en estos territorios y a la inequidad en la obtención de empleo formal, que resulta en un déficit de recursos económicos ya antes mencionados. De lo anterior, Ariza y Hernández (2008) señalan que

"La inequidad en salud se entiende [...] como la presencia de desigualdades en salud, enfermedad y muerte, derivada de una desventaja sistemática de un grupo social respecto de otros como resultado de su posición social. Tal posición resulta de las diferencias de poder, riqueza o prestigio, definidas por su lugar en la estructura laboral o socioeconómica, de género, de raza, de etnia o del ciclo vital en que se encuentra" -Ariza y Hernández, 2008.

De acuerdo con la teoría de Derechos Humanos, las explicaciones de tipo económico sirven para abordar esta problemática, ya que estas hablan de cómo el desarrollo económico y la distribución del capital en las sociedades perpetúan sistemas de desigualdad (Risse-Kappen, et al., 1999). Es decir, que la desigualdad en el aspecto socioeconómico 
entre afrodescendientes y no afrodescendientes, tiene como resultado una menor calidad de vida para los primeros, lo que influye en la falta de obtención de un sistema de salud íntegro y de calidad.

Así mismo, Jiménez (2014) señala que para el periodo entre 1998 y 2002, había una exclusión generalizada que respondía a indicadores de salud bajos, junto a las pocas oportunidades en mercados formales e informales para esta comunidad. En términos de los servicios de salud, existen enormes diferencias entre la población blanca y mestiza, y las comunidades afrocolombianas. Según las estadísticas generales dadas a través del Departamento Nacional de Planeación en ese año, la esperanza de vida de las comunidades afroamericanas se ubicaba $20 \%$ por debajo de la media nacional, que en ese momento era del 71,68\%. El acceso a tales servicios no solo es más restringido para estas comunidades, sino que también enfrentan un mayor déficit en términos de cobertura y calidad de la atención (Jiménez, 2014). Igualmente, existe una desigualdad en el acceso a los servicios de salud cuando estos no reconocen las diferencias o no responden a las necesidades de cada población (Ariza y Hernández, 2008). Si los servicios de salud no se ajustan a los diferentes escenarios correspondientes a las diferentes etnias, y no reconocen las diferentes identidades, están favoreciendo a una población normativamente dominante sobre otra. Así mismo, se debe reconocer lo que cada población entiende por salud y por bienestar para así poder brindar un servicio equitativo. De acuerdo con Muñoz, Puerto, López, Velásquez y Cataño (2014) "Ios grupos tienen formas particulares de definir, nominar y tratar los problemas de salud que no siempre responden a las interpretaciones dadas por los profesionales". Lo anterior significa entonces que los sistemas de salud del país deben empezar a adaptar estrategias culturales al momento de brindar el servicio de salud a fin de reconocer las necesidades existentes desde el punto de vista de las comunidades afrocolombianas y como solucionarlas, teniendo en consideración estos factores culturales. 
En el pacífico, la falta de cobertura en servicios públicos, la ausencia de saneamiento ambiental y el desplazamiento forzado son problemas que ocasionan afecciones en la salud que son en su mayoría prevenibles. Por ejemplo, Muñoz, et al (2014), exponen que "las primeras causas de enfermedad [entre la población afrocolombiana del pacífico] son alteraciones de la salud asociadas a la gestación, hipertensión, infecciones virales, parasitosis, diabetes mellitus, entre otras". Así mismo, enfermedades relacionadas con la desnutrición y afecciones diarreicas agudas son bastante comunes.

Por otra parte, según un estudio realizado por Arrivillaga, Correa, Tovar, Zapata, Varela y Hoyos (2011), la situación de salud es uno de los aspectos más delicados en la región pacífica, en esta zona prevalecen diversas enfermedades tales como la tuberculosis, la desnutrición, el paludismo, la fiebre tifoidea, las enfermedades diarreicas, la infección respiratoria aguda, el cáncer y el VIH/ Sida. En este estudio, se buscaba analizar la presencia de ITS en la población afrocolombiana en la región pacífica, obteniendo como resultados generales que

"el 46.8\% fueron hombres y el 53.2\% mujeres; el 70.4\% tenían escolaridad básica primaria, secundaria y media, el 31.6\% estaba afiliado al régimen contributivo y el $38.5 \%$ al régimen subsidiado, y el $68.2 \%$ residían en cabeceras municipales" -(Arrivillaga et al, 2011).

De lo anterior, se permite decir que existen factores económico-sociales, como la escolaridad y el tipo de afiliación, que influyen directamente en las tasas de personas afrocolombianas que contraen ITS en la región pacífica.

Asimismo, según Díaz (2013, p. 52) se debe buscar, que los Estados no dejen de lado sus obligaciones con respecto a los Derechos Humanos y amparen "el derecho de los pueblos y de los Estados a determinar democráticamente sus políticas alimentarias y agrícolas, sin que estas decisiones vengan dictadas por el sistema comercial internacional". Por lo tanto, es necesario: 
"Proponer que la seguridad alimentaria y nutricional es un estado ideal al que tenemos derecho, y que como tal debe buscarse su garantía, y protegerla, para lo cual es indispensable garantizar a su vez los derechos de soberanía alimentaria, paradigma que bien refleja el fundamental enfoque de derechos humanos" -Díaz, 2013, p. 89.

De igual forma, Díaz (2013) afirma que la malnutrición también genera problemas como la delgadez, sobrepeso y obesidad, todo esto basándose en los indicadores antropométricos de la ENSIN en 2010, en los cuales se distinguen grupos de edad y características socioeconómicas, sociodemográficas y geográficas para poder concluir resultados veraces sobre la situación nutricional del país como aspecto clave en la salud de cada individuo. Del mismo modo, sostiene que "siguen presentándose porcentajes altos en departamentos de regiones con predominio étnico, especialmente relacionado con la mayor prevalencia de los afrocolombianos" y presenta lo anterior a través de un cuadro hecho por la ENSIN (2010), que a través de diferentes índices valora el estado nutricional según la pertenencia étnica y los grupos de población por edad. Concluyendo que la población afro tiene los índices más altos en desnutrición aguda en niños de 0 a 4 años con un porcentaje de 1,6\%. También se encuentra que posee los índices más altos en retraso severo en talla (desnutrición crónica severa) y en delgadez en niños y jóvenes de 5 a 17 años, con porcentajes de 6,3\% y 3\% respectivamente. Por último, en este cuadro se presenta también, los índices más altos en desnutrición (IMC < 18,5) y en obesidad (IMC > 30) en adultos 18 a 64 años, con porcentajes de 3.5\% y 18,2\% respectivamente (2013).

A pesar de la información expuesta con anterioridad, este autor reconoce que los afrocolombianos, como población vulnerable, tienen una relación de invisibilidad frente al Estado con respecto a los demás grupos o comunidades normativas del país, lo cual ha conllevado al fracaso de cualquier mecanismo que busque sofocar la violación a este derecho (Díaz, 2013). No obstante, con base en la información 
recolectada, se permite concluir que la población afrocolombiana en la región pacífica es una comunidad que se encuentra en situación de vulnerabilidad debido a factores étnicos, sociales y económicos, que no permiten que estos tengan un flujo y desarrollo normal de sus derechos.

Problemas que enfrenta la población afrodescendiente del Pacífico colombiano en cuanto al acceso y calidad del sistema educativo.

Hablar de calidad en el sistema educativo involucra múltiples variables que van desde la cobertura, hasta los resultados obtenidos en las pruebas internacionales. Además, es un tema tan extenso que sería necesario desarrollarlo en una investigación aparte. Sin embargo, para poder comparar la situación del pacífico colombiano, es válido hacer un diagnóstico de la situación colombiana en general que, a manera de resumen, es bastante precaria.

"(...) la cobertura educativa ha avanzado de forma importante en los últimos años, aún hace falta completar el esfuerzo para que deje de ser un tema de preocupación. (...) Por otra parte, los indicadores internacionales de aprendizaje muestran que los estudiantes colombianos tienen, en promedio, niveles de aprendizaje comparativamente menores a los de países similares a Colombia. Además, es evidente que existe también una alta inequidad en las horas de instrucción que reciben los estudiantes en nuestro país. Por último, los indicadores de calidad nacionales [muestran] que existen importantes desigualdades en la calidad de la educación al interior del país" -Barrera-Osorio, Maldonado, \& Rodríguez, 2012, p. 2.

Adicionalmente, al tema de la cobertura, se le suma la deserción escolar, la cual es reflejo de dinámicas socioeconómicas y poblacionales estructurales. Este último punto es relevante para el objeto de estudio pues, "en particular, esta se concentra en los hogares de menores ingresos, entre la población rural y los que pertenecen a comunidades étnicas (afrocolombianos e indígenas)" (Delgado, 2014, p. 32).

En ese sentido, se observa que la cobertura en la educación [primaria, secundaria y media] es inferior en el Pacífico que en otras regio- 
nes. En primera instancia, las tasas de cobertura educativa en la región del Litoral Pacífico son del 18,5 \%, mientras que, en la Andina, la cobertura supera el 40\% (Ministerio de Educación Nacional). Respecto al analfabetismo, en algunos municipios del Pacífico como Tumaco esta tasa es del 60\% mientras que, en Cali, por ejemplo, está por debajo del 10\%. Por otra parte, tomando como referencia las pruebas Saber 11 del 2015, existen diferencias entre el puntaje promedio nacional y el obtenido en varios municipios de la región Pacífica. Para ese año el puntaje promedio nacional fue de 255, mientras que las desviaciones de Quibdó, Buenaventura y Tumaco fueron de 30.5, 26.6 y 38.1 respectivamente. Por esta razón, solo el 15\% de los beneficiarios de las becas del Estado, fueron de esta región (Viveros, 2017).

Con relación a la educación superior, la situación no es muy diferente pues también se observan asimetrías en cuanto a la cobertura, por ejemplo;

"El departamento del Valle presenta mayores tasas de estudios de educación superior (T\&T y universitaria) que el Pacífico. Para el caso del Pacífico, esta población presenta tasas muy bajas de asistencia a instituciones de educación superior, para hombres, mujeres, afros y no afros"-Ramírez, Restrepo \& Sabogal, 2015, p. 28.

En este sentido, las condiciones del sistema educativo en el pacífico colombiano respecto a otras regiones del país, comprueban la explicación propuesta por Rodríguez (1977) sobre las grandes desigualdades existentes entre centro y periferia. Según el autor, entre más altos los niveles en la calidad de la educación de las sociedades, mayor será la calidad de los bienes y servicios producidos y a partir de eso, mejor serán los ingresos gracias al intercambio de lo producido. Sería entonces, la ausencia de estas condiciones las que impiden el desarrollo de la periferia. Esta situación es propiciada por el centro, responsable de la distribución de los recursos, el diseño y puesta en marcha de los planes escolares. En este caso, vemos las grandes desigualdades entre el centro -entiéndelo como Cali y la zona Andina- y la periferia 
-entendiéndolo como el departamento del pacífico-, respecto a la diferencia en la cobertura educativa, los resultados en pruebas estatales y analfabetismo. Por ende, lo propuesto por Rodríguez (1977), tendría sentido en la medida que se examine el desarrollo de las mismas, evidenciando un claro avance tanto de Cali como de la zona Andina a diferencia del Pacífico colombiano.

Por otro lado, la Ley 70 de 1993 en el artículo 42 propone la formulación y ejecución de una política destinada a la etnoeducación afrocoIombiana, una promesa sinónimo de protección y reconocimiento. La etnoeducación afrocolombiana es y tiene como propósito:

"resituar a la persona afrodescendiente en un lugar político, social, cultural y económico de dignidad de acuerdo con sus cosmogonías, formas de relacionarse y de estar el universo [...] pretende la liberación y descolonización del sujeto afro ante los más de 500 años de opresión, esclavización y explotación. [Pero también] [...] propende por la educación de todas y todos los individuos de la sociedad colombiana en torno el lugar en la historia nacional de las comunidades afrocolombianas. Además, con un fuerte compromiso con la erradicación de las prácticas racistas y discriminadoras de la sociedad" -Meneses, 2016, p.38.

Sin embargo, en la actualidad es un concepto que se ha dejado de lado y tiene muchos vacíos entre lo que es y no es ser negro, lo que es y no es una comunidad étnica, lo que es y no es la "Cultura afro" formulado por el Estado, y se deja en evidencia la ineficiencia en adoptar las políticas de etnoeducación afrocolombiana. Pues según Meneses (2016):

"la etnoeducación no es solo la incorporación de conocimientos y contenidos en los currículos o en las estructuras del saber del sistema educativo, sino la transformación del conocimiento mismo y las lógicas de producción de este" -(p.38).

En este sentido, se presume que la etnoeducación afrocolombiana en la actualidad es casi inexistente y no ha podido ser estructurada ni 
aplicada correctamente. Así mismo, la calidad no se refiere solamente al contenido que se dicta en las clases, sino también en las variables de insumo y herramientas como el acceso a las TICs o la formación y disponibilidad de docentes, que se ven perturbados en esta región por el contexto o las capacidades de cada municipio, como: falta de energía eléctrica, agua o vías de comunicación. Esto conduce a resultados como los que señala Oscar Sánchez (2018) en su columna de opinión para el periódico El Tiempo: de los 20 municipios de todo el país con peor desempeño en las pruebas Saber para el grado noveno, 14 de los municipios hacen parte del departamento del Chocó y hay un evidente atraso de los estudiantes en los grados escolares, especialmente en las áreas rurales. Sánchez (2018) manifiesta que:

"El diálogo nacional sobre la política de educación étnica para la población afrodescendiente, que lleva años en curso, no parece avanzar. Lo que los colegios ofrecen no está armonizado con el contexto, y entre tanto, la clase política sigue concentrada en la infraestructura y la alimentación escolar, que, como en toda Colombia, han terminado agotando la agenda de la educación" -Sánchez, 2018, párr. 9.

En este último apartado se puede identificar la dinámica de expropiación de recursos planteada por Gunder, Cockcroft \& Johnson (1968), considerando que la metrópoli (el centro) se apropia de los recursos de sus satélites para su propio desarrollo. En el caso de la educación, el recurso que se extrae el pacífico colombiano es el capital humano, pues los que tienen las posibilidades de migrar hacia ciudades con un sistema educativo más robusto, como Cali o la zona Andina, generan desarrollo para ese territorio.

\section{Conclusión}

Con base en la literatura y los datos encontrados, es posible manifestar que los intentos del Estado -desde el artículo transitorio 55 y la posterior Ley 70/93 hasta la actualidad- por proteger, reconocer y dar garantías a las comunidades negras del pacífico colombiano han sido in- 
eficientes e inefectivas. No solo la falta de información sobre estas comunidades y su calidad de vida, sino también la falta de actualización de los datos, dificulta una investigación más profunda; así como también dificulta la formulación y aplicación de políticas acordes al contexto de la región y a las necesidades de la población.

Así mismo, de la información obtenida se encontró que fenómenos y factores como: la violencia, explotación ilegal del territorio, desigualdad, falta de servicios públicos básicos, falta de garantías y altos índices de pobreza, inciden en la constante vulneración de derechos como la salud, el trabajo y la educación de esta población tanto en el territorio como fuera de él. De igual forma, las teorías mencionadas demuestran que las periferias tienden a ser más vulnerables de sufrir los problemas mencionados anteriormente, repercutiendo en violaciones graves de los derechos humanos. Por tales razones, la región pacífica necesita una mayor atención estatal.

Como se ha mostrado, los tres derechos que se exponen en este artículo -trabajo, salud y educación- tienen una correlación entre sí. Es necesario una mejor calidad en la educación para encontrar y acceder a mejores oportunidades de empleo, pero también es necesario mejores oportunidades de empleo para acceder a un mejor sistema de salud, para asegurar una mejor calidad de vida en las comunidades negras que así mismo pueda incidir en el acceso a la educación y el trabajo.

Los resultados de esta investigación permiten afirmar que la población afrocolombiana de la región pacífica tiene menos probabilidades de conseguir o acceder a empleos de calidad y tienen menos oportunidades laborales en comparación al resto del país y la población afrocolombiana fuera de ese territorio. Así mismo, se concluye que el hecho de que no puedan acceder a trabajos de calidad en su territorio los obliga a desplazarse a otras ciudades o regiones del país, construyendo y alimentando la dinámica centro-periferia explicada en las discusiones, pues los trabajos que se encuentran en la región no garantizan 
una calidad de vida digna. Igualmente, lo expresado en la discusión nos lleva a concluir que las personas negras no solo tienden a trabajar en empleos de carácter informal o de baja calidad, sino que también tienen menos estabilidad que el resto de la población colombiana porque son los únicos trabajos que les quedan ante la falta de oportunidades.

Con respecto al ámbito de la salud, la teoría de los DD.HH. se enfoca en los aspectos claves con respecto al área de la salud. Sabemos que, si bien se han hecho esfuerzos para visibilizar cada vez más a la población afrodescendiente, a través de una mayor inclusión en los planes de desarrollo gubernamentales, encontramos que el panorama no es alentador.

Por un lado, se encuentran tasas elevadas de malnutrición, lo cual a su vez deriva en una reducción de los estándares de vida. Por otro lado, se encuentra la situación de vulnerabilidad en la que se halla esta población con respecto a las enfermedades, que como se mencionaron anteriormente, tienden a ser: tuberculosis, desnutrición, paludismo, fiebre tifoidea, enfermedades diarreicas, cáncer y el VIH/ Sida. Teniendo en cuenta el contexto que presentan los afrocolombianos como población en situación de vulnerabilidad étnica, social y económica, se evidenció que no solo son más propensos a sufrir estas enfermedades, sino que también son menos las oportunidades de recibir atención médica de calidad.

Respecto a la educación, al comparar la región del Pacífico con el resto del país en esta materia, existen diferentes desigualdades que van desde la cobertura hasta los resultados obtenidos en las pruebas nacionales. En este sentido, mientras que en los centros del país la cobertura llega a ser superior al 40\%, en el Pacífico esta meramente alcanza el 18\%. De igual forma, la situación es similar en la educación superior; son pocos los afrodescendientes de esta región que logran ingresar a universidades públicas o privadas. 
Así mismo, se identificaron algunos elementos teóricos en cuanto a la dinámica centro-periferia para la situación del sistema educativo en la región del Pacífico. La calidad de la educación, por una parte, no permite el desarrollo de sistemas productivos con valor agregado que generen ingresos a la región y con ello, diseñar y aplicar políticas públicas que generen bienestar en la población. Por otra parte, el centro expropia recursos humanos valiosos que podrían ser de gran ayuda para el pacífico, puesto que, ante las diferencias en calidad del pacífico frente a otras regiones centrales, individuos que tienen la posibilidad de educarse en esas zonas generan a futuro desarrollo para las metrópolis.

Otro factor destacable es la deserción escolar, que normalmente es más común en ambientes con dinámicas socioculturales violentas y pobres. Teniendo en cuenta que los afrodescendientes han sido víctimas históricas de estas problemáticas, se encuentra una relación entre estas dinámicas y que sea esta población en la que se evidencian tasas más altas de deserción. En cuanto al desempeño de los estudiantes, se evidencia que, en las pruebas de Estado ICFES, la región del pacífico tuvo resultados mucho más bajos que el promedio nacional.

Finalmente, sería importante que a partir de esta investigación no solo se anime a investigar más sobre la evidente segregación que sufre esta población en la actualidad, sino también que se formulen y apliquen políticas públicas o medidas para evitar y mitigar las violaciones a los derechos humanos de las comunidades negras del pacífico. Lo anterior, para garantizar la igualdad, equidad, vida digna y oportunidades de calidad que se consagró en la constitución colombiana acordes a las verdaderas realidades de la región pacífica y los contextos de la población. Es un tema que debería ser más relevante a escala de estudio nacional y es prioritaria la recolección de datos y cifras actualizadas para intentar solventar las problemáticas a las que se enfrenta esta población. 


\section{Bibliografía}

Amnistía Internacional. (2001, febrero). Recomendaciones de Amnistía Internacional a los gobiernos con ocasión de la Conferencia Mundial contra el Racismo, la Discriminación Racial, la Xenofobia y las Formas Conexas de Intolerancia (41/002/2001/s). https://www. amnesty.org/download/Documents/128000/ior410022001es.pdf

Ariza-Montoya, J. F., \& Hernández-Álvarez, M. E. (2008). Equidad de Etnia en el Acceso a los Servicios de Salud en Bogotá, Colombia, 2007. Revista de Salud Pública, 10, 58-71. https://doi.org/10.1590/ s0124-00642008000600006

Arrivallaga, M., Correa, D., Tovar, L. M., Zapata, H., Varela, M. T., \& Hoyos, P. A. (2011). Infecciones de transmisión sexual en la región Pacífica colombiana: implicaciones para población en situación de vulnerabilidad étnica, social y económica. Pensamiento Psicológico, 9(16), 145-162. http://www.scielo.org.co/scielo.php?script=sci_ arttext\&pid=S1657-89612011000100010\&lng=en\&tlng=es

Ayala Osorio, G., Flórez, J., Hurtado, G., \& Lobato, L. (2014). Ethos Regiona: Boletín del Centro Interdisciplinario de Estudios de la Región Pacífico Colombiana (N.o 11). Centro Interdisciplinario de Estudios de la Región Pacífico Colombiana, CIER. https://www.uao.edu.co/ sites/default/files/Ethos\%20Regional\%2011a\%20edici\%C3\%B3n. pdf

Barrera-Osorio, F., Maldonado, D., \& Rodríguez, C. (2012). Calidad de la educación básica y media en Colombia: diagnostico y propuestas (N.o 126). Universidad del Rosario: Facultad de Economía. https:// repository.urosario.edu.co/bitstream/handle/10336/10907/10078. pdf

Congreso de la República, Ley 70 del 27 de agosto de 1993 "Por la cual se desarrolla el artículo transitorio 55 de la Constitución Política" en Diario Oficial No. 41.013. Agosto 31 de 1993. 
Correa, N. (2018). Iniciativas públicas y privadas a favor de la pobreza rural indígena y afrodescendiente en América Latina: balance y perspectiva. Organización de las Naciones Unidas para la Alimentación y la Agricultura. https://repositorio.iep.org.pe/handle/ IEP/1145

Defensoría del Pueblo. (2016). Problemática humanitaria en la región pacífica colombiana (N.o 1). https://www.defensoria.gov.co/ public/pdf/Informepacificoweb.pdf

Delgado Barrera, M. (2014). LA EDUCACIÓN BÁSICA Y MEDIA EN COLOMBIA: RETOS EN EQUIDAD Y CALIDAD. FEDESARROLLO. https://www.repository.fedesarrollo.org.co/bitstream/ handle/11445/190/La\%20educaci\%c3\%b3n\%20b\%c3\%a1sica\%20 y\%20media\%20en\%20Colombia\%20retos\%20en\%20equidad\%20 y\%20calidad\%20-\%20KAS.pdf?sequence=2\&isAllowed=y

Departamento Nacional de Estadística. (2018-2020). Boletín Técnico Gran Encuesta Integrada de Hogares (GEIH) (Mercado laboral por regiones Segundo semestre 2019) [Conjunto de datos]. DANE. https://www.dane.gov.co/files/investigaciones/boletines/ech/ech_ regiones/bol_regiones_lls_19.pdf

Departamento Nacional de Estadística (DANE). (2019). POBLACIÓN NEGRA, AFROCOLOMBIANA, RAIZAL Y PALENQUERA: RESULTADOS DEL CENSO NACIONAL DE POBLACIÓN Y VIVIENDA 2018. https://www.dane.gov.co/files/investigaciones/boletines/grupos-etnicos/presentacion-grupos-etnicos-poblacionNARP-2019.pdf

Díaz, J. (2013). Inseguridad alimentaria en la población indígena y afrodescendiente en Colombia. [Tesis de Máster, Universidad de Barcelona] Recuperado de: http://diposit.ub.edu/dspace/handle/2445/55530

Di Filippo, A. (1998). La visión centro-periferia hoy. Revista CE- 
PAL, RCEX01(Extraordinario), 175-185. https://repositorio.cepal. org/handle/11362/12135

Donelly, J. (2003). La construcción social de los derechos humanos. Relaciones internacionales: Revista académica cuatrimestral de publicación electrónica, 17, 153-184. https://dialnet.unirioja.es/ servlet/articulo?codigo=3678194

Fraser, N. (1998). Nancy Fraser. (1997). Lustitia Interrupta. Reflexiones críticas desde la posición «postsocialista». Bogotá: Siglo del Hombre Editores, Uniandes. Revista de Estudios Sociales, 2, 150151. https://doi.org/10.7440/res2.1998.29

Galvis-Aponte, L. A., \& Pérez-Valbuena, G. J. (2015). Documentos de Trabajo Sobre Economía Regional: Informalidad laboral y calidad del empleo en la Región Pacífica colombiana (N.o 233). Banco de la Republica - Centro de Estudios Regionales. https://www.banrep.gov. co/sites/default/files/publicaciones/archivos/dtser_233.pdf

González de Dios, J., \& Buñuel Álvarez, J. C. (2006). Búsqueda eficiente de las mejores pruebas científicas disponibles en la literatura: fuentes de información primarias y secundarias. EVIDENCIAS EN PEDIATRIA, 2(1), 1-10. https://evidenciasenpediatria.es/articulo/5026/busqueda-eficiente-de-las-mejores-pruebas-cientificasdisponibles-en-la-literatura-fuentes-de-informacion-primarias-y-secundarias

Goodale, M., \& Merry, S. E. (2007). The Practice of Human Rights. Cambridge University Press.

Gunder, F., Cockcroft, J., Johnson, D. (1968)- Economía política del subdesarrollo en América Latina. Recuperado de: http://www.archivochile.cl/Ideas_Autores/gunderfa/gunderfa0006.pdf

Gunder, F. (1977). El desarrollo y el subdesarrollo. Recuperado de: https://repositorio.cepal.org/handle/11362/44455 
Hernández Sampieri, R., Fernández Collado, C., \& Baptista Lucio, P. (1997). METODOLOGÍA DE LA INVESTIGACIÓN [Libro electrónico]. https://www.uv.mx/personal/cbustamante/files/2011/06/ Metodologia-de-la-Investigaci\%C3\%83\%C2\%B3n_Sampieri.pdf

Krause, M. (1995). La investigación cualitativa: un campo de posibilidades y desafíos. Revista Temas de educación, 7(7), 19-40. https:// www.researchgate.net/publication/215561167_La_investigacion_ cualitativa_Un_campo_de_posibilidades_y_desafios

Meneses, Y. (2016). La etnoeducación afrocolombiana: conceptos, trabas, patriarcado y sexismo. A propósito de los 20 años de la Ley General de Educación 115 de 1994. Revista Historia De La Educación Latinoamericana, 18(27), 35-66. https://doi. org/10.19053/01227238.5508

Monje, C. (2011). Metodología de la Investigación Cuantitativa y Cualitativa: Guía Didáctica (pp. 12-13). Universidad Surcolombiana. Tomado de: https://www.uv.mx/rmipe/files/2017/02/Guia-didactica-metodologia-de-la-investigacion.pdf

Muñoz, A. (2008). TEORÍA DE LOS DERECHOS HUMANOS, LOS DERECHOS DE LAS MUJERES Y EL DERECHO A LA EDUCACIÓN. Conceptos y desarrollo de los mismos en el Discurso Internacional. UNIVERSIDAD DE SALAMANCA. PROGRAMA DE DOCTORADO «PASADO Y PRESENTE DE LOS DERECHOS HUMANOS». https:// gredos.usal.es/bitstream/handle/10366/21673/DHMMC_Teoria\%20Derechos.pdf;jsessionid=DA2E93002E3C490ADF51D3EFC 6550FE1? sequence $=1$

Muñoz, A. I., Puerto, A. H., López, L., Velásquez, V. F., \& Cataño, N. (2014). Perceptions of health-illnes and ways of care in Afro-Colombian vulnerable families. Index de Enfermería, 23(4), 214-218. https://doi.org/10.4321/S1132-12962014000300005

Organización Internacional para las Migraciones (OIM). (2015). 
Una paz estable, duradera y sensible a niños, niñas, adolescentes y jóvenes Chocó. https://repository.oim.org.co/bitstream/handle/20.500.11788/1638/86.5.\%20COLOIM\%200218\%20V5\%20 Choco.pdf? sequence $=9$ \&isAllowed $=y$

Pérez, I. (2015, 20 marzo). Racismo, causa de sufrimiento de millones de personas. DGDCUNAM. http://ciencia.unam.mx/leer/441/ racismo_causa_de_sufrimiento_de_millones_de_personas

Pérez, L., \& Mora, J. J. (2015). La calidad del empleo en la población afrodescendiente colombiana: una aproximación desde la ubicación geográfica de las comunas. Revista de Economía del Rosario, 17(2), 315-347. https://www.urosario.edu.co/economia/documentos/ VE17n2_Perez.pdf

Ramírez, J. M., Martínez-Restrepo, S., \& Sabogal, A. (2015). Educación Técnica y Tecnológica En El Pacífico colombiano: un camino para el desarrollo y la inclusión social (1.a ed.) [Libro electrónico]. Fundación FEDEDESARROLLO. https://www.repository.fedesarrollo.org.co/bitstream/handle/11445/2668/EducacionTecnicayTecnologicaenelPacificoColombiano.pdf? sequence=1\&isAllowed=y

Rangel, M., \& Del Popolo, F. (2011). Juventud afrodescendiente en América Latina: realidades diversas y derechos (in)cumplidos. Economic Commission for Latin America and the Caribbean. http:// equidadparalainfancia.org/2013/08/juventud-afrodescendiente-enamerica-latina-realidades-diversas-y-derechos-incumplidos/

Reales, L. (2014). Informe del Movimiento Nacional Afrocolombiano CIMARRÓN sobre la situación de derechos humanos de la población afrocolombiana (1994-2004). Bogotá: Junta Directiva del Movimiento CIMARRÓN. https://www.urosario.edu.co/urosario_ files/04/04415217-2689-4ba7-8699-4b5a16c0bf71.pdf

Risse, T., Ropp, S., \& Sikkink, K. (1999). The power of human rights: 
International norms and domestic change (Vol. 66). Cambridge University Press. https://books.google.es/books?hl=es\&lr=\&id=kpsDP vaCOCAC\&oi=fnd\&pg=PR9\&dq=katheryn+sikink+\&ots=nc344r l 3_s\&sig=rHOhFec066nkVxIrunHOcWwRs\#v=onepage\&q=kather yn\%20sikink\&f=false

Rodríguez, C., Cárdenas, J. C., Oviedo, J. D., \& Villamizar, S. (2013). La discriminación racial en el trabajo Un estudio experimental en Bogotá. Centro de Estudios de Derecho, Justicia y Sociedad, Dejusticia. https://economia.uniandes.edu.co/files/profesores/juan_camiIO_cardenas/docs/Archivos\%20para\%20descargar/ODR__DISCR_ TRABAJO-small.pdf

Rodríguez, O. (1977). Sobre la concepción del sistema centroperiferia. Revista de la CEPAL. https://repositorio.cepal.org/handle/11362/12422

Sánchez, O. (2018, 14 noviembre). Educación en el Chocó: La guerra y la desconfianza en el Estado, factores profundos de la debilidad educativa. El Tiempo. https://www.eltiempo.com/opinion/columnistas/oscar-sanchez/educacion-en-el-choco-columna-de-oscarsanchez-293414

Vera, J. (2008). Razas y Racismo: entre la unidad y la diversidad de nuestra especie. https://www.revistaciencia.amc.edu.mx/images/ revista/59_2/PDF/08-617-52-.pdf

Viveros, V. (2017, 26 mayo). La Educación en el Pacífico es el camino. La Silla Llena. https://lasillavacia.com/silla-llena/red-pacifico/historia/la-educacion-en-el-pacifico-es-el-camino-61099

Wade, P. (1993). La relación Chocó-Antioquia: Un caso del colonialismo interno. Vol. 2. Fondo Energético Nacional. https://www. academia.edu/34116263/La_relaci\%C3\%B3n_Choc\%C3\%B3-Antioquia_Un_caso_del_colonialismo_interno 